Preface

\title{
Editorial Compilation VI
}

\author{
Emmanuel J. Favaloro, PhD, FFSc (RCPA) ${ }^{1}$ Giuseppe Lippi, MD ${ }^{2}$ \\ ${ }^{1}$ Department of Haematology, Institute of Clinical Pathology and \\ Medical Research (ICPMR), Sydney Centres for Thrombosis and \\ Haemostasis, Westmead Hospital, Westmead, Australia \\ 2 Section of Clinical Biochemistry, University of Verona, Verona, Italy \\ Semin Thromb Hemost 2019;45:5-9.
}

Welcome to the latest issue of Seminars in Thrombosis and Hemostasis (STH) published under the "banner" of "Editorial Compilation," this being the sixth such issue. Although STH is primarily a theme-driven publication, an ongoing opportunity arises to publish some issues containing more wideranging chapters of current interest and controversy, which do not quite match a current themed issue in progress. We also require a medium to enable publication of contributions from our Eberhard F. Mammen Young Investigator Award winners. ${ }^{1-7}$ As now standard for this compilation series, the current issue has a mixture of content that comprises all these elements, as well as broadly fitting within the separate themes of "thrombosis" and "bleeding."

This issue begins with some contributions related to improving laboratory diagnostics, ${ }^{8,9}$ and representing the start of a new series of "how to..." articles for STH. The first paper provides suggested approaches to generating a more accurate laboratory-based international normalized ratio (INR), including solutions to obtaining or verifying the mean normal prothrombin time (MNPT) and international sensitivity index (ISI). ${ }^{8}$ Although the landscape of anticoagulation therapy is evolving, vitamin $\mathrm{K}$ antagonists (VKAs) such as warfarin remain anticoagulants of choice for many clinicians and their patients. ${ }^{10}$ Nevertheless, management of VKA therapy remains challenging, largely because of individualized patient metabolism and drug and food interactions, which means that VKA dosing has to be personalized. This is achieved by regular patient monitoring using the prothrombin time (PT), as mathematically converted to INR. ${ }^{11}$ The INR test represents the most often employed routine hemostasis test, and is meant to harmonize laboratory test results by taking into account reagent and instrumentation variability, which is otherwise expected to give rise to variable PT values, but which should accordingly lead to less variable INR values. Of clinical importance, too low an INR is suggestive of increased thrombotic risk and typically means the VKA

Address for correspondence Emmanuel J. Favaloro, PhD FFSc (RCPA), Department of

$\begin{array}{ll}\text { (RCPA), Department of } & \text { Emmanuel J. Favaloro, PhD, FFSC } \\ \text { Haematology, Institute of Clinical } & \text { (RCPA), and Giuseppe Lippi, MD. }\end{array}$ Pathology and Medical Research (ICPMR), Westmead Hospital,

Westmead, Australia

(e-mail: emmanuel.

favaloro@health.nsw.gov.au).
Compilation VI; Guest Editors:

dose should be increased, whereas too high an INR is suggestive of increased bleeding risk and typically means the VKA dose should be temporarily withheld and/or decreased. However, evidence continues to show that variability in INR values between laboratories remains unacceptably high. ${ }^{12,13}$ Given that modern instrumentation provides for robust analytical values-meaning highly reproducible intralaboratory clotting times or PTs in this case-the most likely cause of high INR variability is inconsistency in the INR test components-meaning the MNPT and ISI values used by laboratories to generate a given INR. ${ }^{14}$ In other words, there are doubts as to the accuracy of some INR values, because there are corresponding doubts about the accuracy of MNPT and/or ISI values that have been assigned by some laboratories for their reagent/instrument combination. ${ }^{14}$ The current report is, therefore, intended to provide some solutions around the problems of inaccurate INRs, ISIs, and MNPTs, thus aiming to drive laboratory INRs closer to "truth," improving harmonization/standardization, and finally promoting better patient management. There are several novel strategies outlined, including a primary process of transference to obtain/verify MNPT and/or ISI values for a new reagent using an existing reagent as reference, and a secondary process whereby External Quality Assessment data can be used to correct bias or existing errors in assigned MNPT and/or ISI values.

Next is a similar contribution around the second most popular routine hemostasis assay, the activated partial thromboplastin time (aPTT), which may be used by clinicians for several reasons. ${ }^{9}$ The test is conventionally used for assessing the contact factor (intrinsic) pathway of blood coagulation, and thus for screening deficiencies in this pathway, most typically factors (F)VIII, FIX, and FXI. The aPTT is also sensitive to contact factor deficiencies, including FXII, prekallikrein, and high molecular weight kininogen. The aPTT may also be increased in a variety of conditions,

Copyright $\odot 2019$ by Thieme Medical Publishers, Inc., 333 Seventh Avenue, New York, NY 10001, USA. Tel: +1(212) 584-4662.
DOI https://doi.org/ 10.1055/s-0038-1677462.
ISSN 0094-6176. 
including liver disease, vitamin $\mathrm{K}$ deficiency, and disseminated intravascular coagulation (DIC). The aPTT can also be used for monitoring unfractionated heparin (UFH) therapy, as well as for screening lupus anticoagulant (LA) or for assessing thrombosis risk. ${ }^{15,16}$ Which of these separate uses is important to a given laboratory or clinician depends on the laboratory and the clinical context. For example, UFH sensitivity is important in hospital-based laboratories, where UFH therapy may be used, but is not important in hospital-based laboratories where low-molecular-weight heparin (LMWH) is largely employed or where UFH may be assessed by anti-FXa testing, or in private/community laboratories not associated with a hospital system. High sensitivity to (low levels of) FVIII, FIX, and FXI is generally preferred, since their deficiencies are clinically significant. Also preferred, but not usually achieved, is low sensitivity to FXII and other contact factors, since patients expressing these deficiencies are typically asymptomatic. Nevertheless, a good knowledge of factor sensitivity is usually needed, if only to help explain the reasons for a prolonged aPTT in a given patient, or whether factor testing or other investigations are required. A good working knowledge of reagents sensitivity to LA is also advisable, especially when the reagent is used as part of a LA test panel, or else as a "general-purpose screening reagent." This report is aimed at providing some guidance around these questions, intended as a kind of "how to" guide that will enable laboratories to assess aPTT reagents in regard to their sensitivity to heparin, LA, and clotting factors. The report also provides some advice on generation of normal reference intervals, as well as solutions for troubleshooting prolonged aPTTs, and when to perform factor testing or search for inhibitors.

This issue of STH continues the exploration of laboratory methodologies with the third contribution, by Stufano et al, on an evaluation of the utility of the von Willebrand factor (VWF) propeptide (VWF:pp) assay in the differential diagnosis of von Willebrand disease (VWD) and acquired von Willebrand syndrome (AVWS). ${ }^{17}$ An increased VWF:pp to VWF antigen (VWF:Ag) ratio (VWF:pp/VWF:Ag) indicates an enhanced plasma clearance of VWF. A distinction between congenital VWD and AVWS is primarily based on family and personal history of bleeding. If this information is sparse, however, the diagnosis might be challenging due to the lack of an effective diagnostic biomarker. In this cross-sectional study, the authors assessed the capacity of VWF:pp/VWF:Ag to guide the differential diagnosis between VWD and AVWS. VWF:pp/VWF:Ag was measured in a group of 153 patients (125 with VWD and 28 with AVWS). Most patients with AVWS and VWD showed an increased VWF:pp/VWF:Ag, although to variable degrees. A marked increase in VWF: pp/VWF:Ag was mainly associated with the diagnosis of AVWS and VWD type 1 Vicenza. This report, therefore, adds to the diagnostic repertoire of VWD and AVWS. ${ }^{18}$

Continuing the exploration of bleeding disorders, Dorgalaleh et $\mathrm{al}^{19}$ review some genetic and clinical features of FXIII deficiency, as well as presenting some original data on Khash FXIII-being a unique FXIII gene (F13A) mutation with unex- pectedly high prevalence in southeastern Iran. Congenital FXIII deficiency is an extremely rare hemorrhagic disorder associated with a high rate of morbidity and mortality, including a high rate of intracranial hemorrhage. ${ }^{20}$ The disorder is more frequent in Iran, especially in Khash, a city in the southeast of the country. As identified in the current report, the prevalence of FXIII deficiency in this city is 1 homozygote per $\sim 500$ population (which is $\sim 4,000$ times higher than the worldwide prevalence), with $3.5 \%$ heterozygotes. The disorder is accompanied by a high rate of mortality in rural areas of Khash, given an averaged observed rate of $\sim 3$ deaths per each family with FXIII deficiency, mostly due to late-diagnosis and/ or misdiagnosis, and fetal consequences of both umbilical cord and central nervous system bleeding. Almost all patients with FXIII deficiency in the southeast Iran have a unique mutation in the F13A gene (Trp187Arg), which results in a severe FXIII deficiency. This mutation is used for premarriage and prenatal diagnosis, as well as for carrier detection and diagnostic confirmation. In conclusion, this report provides novel insights into severe FXIII deficiency, its diagnosis, prevalence, molecular basis, clinical manifestations, management, and treatment, with particular focus on Iran, representing a hotspot for this disorder. This contribution can be seen to also complement other recent papers in this journal on FXIII deficiency and treatment. ${ }^{20,21}$

We move this issue of STH from bleeding disorders to prothrombotic considerations with the next contribution from Arachchillage and Makris, ${ }^{22}$ who ask the question: "Inherited thrombophilia and pregnancy complications: should we test?." Recurrent miscarriages and pregnancyrelated complications cause significant stress to couples looking for successful pregnancy outcomes, as well as to the healthcare professionals who care for them. There is conflicting evidence with respect to presence and strength of associations between inherited thrombophilia and these complications. A complete thrombophilia screen is expensive. There is no proven currently available and effective treatment for women with recurrent miscarriage and inherited thrombophilia. Based on the concept of microvascular thrombosis of placenta, women with recurrent miscarriage and placenta-related complications frequently get treated with antithrombotic therapy. In this narrative review, the authors explore the evolving understanding and evidence of inherited thrombophilia in recurrent miscarriage and other pregnancy complications, and whether antithrombotic treatment would modify pregnancy outcome in women with inherited thrombophilia. Finally, the authors provide some personal recommendations based on available evidence for clinical practice. In summary, the authors propose that inherited thrombophilia testing is not required for women with recurrent pregnancy losses or late pregnancy complications outside the setting of a clinical trial. Moreover, the presence of thrombophilia markers does not generally indicate additional therapy during pregnancy, even if a heritable thrombophilic defect is found in women with recurrent miscarriages or late pregnancy complications.

Continuing the discussion around prothrombotic disorders and pregnancy morbidity, Kubisz et al provide an update 
review $^{23}$ on the condition known as "Sticky Platelet Syndrome" on the 35th anniversary of its initial description, and which is accompanied by a Commentary. ${ }^{24}$ In support of the previous review, ${ }^{22}$ these authors propose that past research into "thrombophilia" has predominantly focused on coagulation factors and not on other components of the hemostatic system. The possible role of platelet function defects in the development of thrombotic events was initially suggested in the late 1970s, when an increased platelet adhesiveness and aggregation after epinephrine and adenosine diphosphate (ADP) were reported in patients with unexplained transient ischemic attack. Clinical evidence for other types of thrombotic events (e.g., myocardial infarction, ischemic stroke, optic neuropathy, and pregnancy-related complications) with similar laboratory findings was later provided by several authors in the 1980s and 1990s. Familial occurrence was also noted, and the term "sticky platelet syndrome" was introduced in 1983 to describe the defect. The term, in present understanding, describes a thrombophilic qualitative platelet disorder characterized by increased in vitro platelet aggregation after addition of very low concentrations of ADP and/or epinephrine along with an increased risk of thromboembolic (predominantly arterial) events. Although now recognized for nearly 35 years, significant issues, namely its etiology, inheritance, epidemiology, and diagnostics, remain matters of vigorous debate. Accordingly, the aim of the present review is to summarize the history, key works, and present understanding of the syndrome and outline present-day diagnostic and clinical problems and controversies. ${ }^{23}$

This issue of STH then moves from potential pregnancyrelated complications, to monitoring antiplatelet therapy in neonates and children, within a systematic review from Huang et al. ${ }^{25}$ As well recognized by our readership, antiplatelet agents are used for prevention and treatment of thromboembolic disease. This review, therefore, focuses on the increasingly complex population of pediatric patients. Despite the importance of treatment for clinical outcome, there is no consensus on the most effective monitoring strategies. The authors searched five medical publication databases from 2000 to 2017, using keywords selected a priori, and sorted articles according to the antiplatelet agents administered, methods of antiplatelet monitoring, and outcome measures. Twenty studies were included in the systematic review, comprising fourteen cohort studies, three randomized controlled trials, and three cross-sectional investigations. Eleven different antiplatelet monitoring tools were used, with the most common being Light Transmission Aggregometry, Urinary Thromboxane, Thromboelastography with Platelet Mapping, and VerifyNow. In the majority of studies, antiplatelet therapy monitoring was used to describe adequacy or responsiveness to treatment based on laboratory cut-off values, which were not uniform and sourced from adult studies or extrapolated from test manuals. Several studies evaluated monitoring related to clinical outcome or adjusted therapy to reach predefined therapeutic targets. There was no single laboratory method found to be distinctly better for monitoring antiplatelet treatment. Asso- ciations between laboratory assays and clinical outcomes or assays and gold-standard measurements were highly inconsistent. The authors conclude by indicating that the current literature lacks consensus on clinical benefits and measurable effects of monitoring antiplatelet therapy in pediatric patients. The review also highlights important areas for research needed to determine the value of antiplatelet therapy monitoring in children.

The concept of thrombosis continues with the next issue contribution, as prepared by a recent Eberhard Mammen Young Investigator Award winner, Laridan et al, on neutrophil extracellular traps (NETs) in arterial and venous thrombosis. $^{26}$ As discussed by these authors, thrombotic complications remain a major health risk worldwide. However, viewpoints on the pathophysiology of thrombosis have significantly changed since the discovery of NETs and their prothrombotic characteristics. Generated by neutrophils that release their decondensed chromatin as a network of extracellular fibers, NETs promote thrombus formation by serving as a scaffold that activates platelets and coagulation. The thrombogenic involvement of NETs has been described in various settings of thrombosis, including stroke, myocardial infarction, and deep vein thrombosis. The aim of this particular review is to summarize existing evidence showing the presence of NETs in human thrombus material. Following an introduction on NETs and their role in thrombus formation, the authors address studies showing the presence of NETs in arterial or venous thrombi. In addition, the authors focus on potential novel therapeutic opportunities to resolve or prevent thrombosis by targeting NETs.

Another contribution around the concept of thrombosis treatment ensues with the original study from Maclachlan et al, this time in relation to obesity. ${ }^{27}$ Obese patients are under-represented or excluded in previous trials assessing venous thromboembolism (VTE) treatment. ${ }^{28}$ The authors have, therefore, examined the safety of weight-based LMWH (enoxaparin) dosing in obesity, as assessed by antiactivated Factor X (anti-FXa) activity, bleeding, and recurrence. They performed a 5-year retrospective audit of patients with acute VTE, weighing $>100 \mathrm{~kg}$, prescribed enoxaparin $1 \mathrm{mg} / \mathrm{kg}$ twice daily, with an anti-FXa level 2 to 6 hours post-dose. The primary outcome was anti-FXa levels, and the secondary outcomes were bleeding and recurrence. Results were compared with patients weighing $<100 \mathrm{~kg}$ and obese patients prescribed doses $<1 \mathrm{mg} / \mathrm{kg}$. The median peak anti-FXa level was $0.93 \mathrm{U} / \mathrm{mL}$, with $56 \%$ of levels being in the proposed therapeutic range $(0.5-1.0 \mathrm{U} / \mathrm{mL}), 40 \%>1.0 \mathrm{U} / \mathrm{mL}$, and $4 \%<0.5 \mathrm{U} / \mathrm{mL}$. The median anti-FXa levels and distribution were not significantly different between patients $>100 \mathrm{~kg}$ and patients $<100 \mathrm{~kg}$, while obese patients prescribed $<1$ $\mathrm{mg} / \mathrm{kg}$ were more frequently subtherapeutic (21\%). Regardless of weight, the majority of patients with moderate renal impairment (eGFR 30-59 mL/min) had an anti-FXa level $>1.0 \mathrm{U} / \mathrm{mL}$ (61\%). In the obese patients, there was no major bleeding or recurrence within 30 days. In comparison, patients weighing $<100 \mathrm{~kg}$, despite similar anti-FXa peak levels, had higher rates of bleeding and recurrence. However, this was likely due to their older age and comorbidities, 
particularly renal impairment and cancer. The authors conclude that these data support weight-based dosing of enoxaparin in obesity with no maximum dose, ensuring therapeutic drug levels, with anti-FXa levels suggested in obese patients with clinical risk factors for bleeding.

The final full-length contribution to this issue of STH is from Thachil, who asks the question: "Does a diagnosis of DIC exist anymore?" ${ }^{29}$ DIC is recognized to represent an intermediary mechanism of disease known to develop as a complication in other conditions like sepsis, trauma, cancer, or obstetrical disorders. Thus, patients with DIC may present to different specialists depending on symptomology, and a good understanding of the pathophysiological process is, hence, necessary to ensure best management. However, more recently, controversy has risen where experts doubt whether DIC really exists in many of the historically well-established diagnoses. This has led to confusion among both basic science researchers and clinical practitioners about when to consider DIC diagnosis. In this review, the various issues which have led to this uncertainty are addressed, including the problem with different terminologies, simpler explanation of current DIC diagnostic criteria, reasons behind why the diagnosis may be overlooked or not considered at all, along with their possible solutions. The author hopes that the diagnostic aspects of DIC will come full circle, wherein the recent research can build on what history had taught and not to refute its existence.

As usual for these nonthematic issues of STH, this issue also has some correspondence, ${ }^{30-32}$ including an unusual case of FV deficiency associated with thrombosis. We also include in this issue of STH, somewhat less typically, a meeting report ${ }^{33}$ and also an Editorial. ${ }^{34}$ Finally, we thank all the authors to this latest issue of "Editorial Compilations" for their original and comprehensive contributions, and we hope our readership enjoys this "bumper" issue of STH as much as we did in compiling same.

\section{References}

1 Favaloro EJ. 2011 Eberhard F. Mammen award announcements. Semin Thromb Hemost 2011;37(05):431-439

2 Favaloro EJ. 2012 Eberhard F. Mammen award announcements. Semin Thromb Hemost 2012;38(05):425-432

3 Favaloro EJ. 2013 Eberhard F. Mammen award announcements. Semin Thromb Hemost 2013;39(06):567-574

4 Favaloro EJ. 2014 Eberhard F. Mammen award announcements: part II-Young Investigator Awards. Semin Thromb Hemost 2014; 40(07):718-723

5 Favaloro EJ. 2015 Eberhard F. Mammen award announcements: part II-Young Investigator Awards. Semin Thromb Hemost 2015; 41(08):809-815

6 Favaloro EJ. 2016 Eberhard F. Mammen award announcements: part II-Young Investigator Awards. Semin Thromb Hemost 2017; 43(03):235-241

7 Favaloro EJ. 2017 Eberhard F. Mammen award announcements: part II-Young Investigator Awards. Semin Thromb Hemost 2018; 44(02):81-88

8 Favaloro EJ. How to generate a more accurate laboratory-based international normalized ratio (INR): solutions to obtaining or verifying the mean normal prothrombin time (MNPT) and inter- national sensitivity index (ISI). Semin Thromb Hemost 2019;45 (01):10-21

9 Favaloro EJ, Kershaw G, Mohammed S, Lippi G. How to optimize activated partial thromboplastin time (APTT) testing: solutions to assessing normal reference ranges and APTT reagents for sensitivity to heparin, lupus, and clotting factors. Semin Thromb Hemost 2019;45(01):22-35

10 Favaloro EJ, Pasalic L, Lippi G. Replacing warfarin therapy with the newer direct oral anticoagulants, or simply a growth in anticoagulation therapy? Implications for pathology testing. Pathology 2017;49(06):639-643

11 Favaloro EJ. Optimizing the Verification of Mean Normal Prothrombin Time (MNPT) and International Sensitivity Index (ISI) for Accurate Conversion of Prothrombin Time (PT) to International Normalized Ratio (INR). Methods Mol Biol 2017; 1646:59-74

12 Kitchen DP, Jennings I, Kitchen S, Woods TA, Walker ID. Bridging the gap between point-of-care testing and laboratory testing in hemostasis. Semin Thromb Hemost 2015;41(03): $272-278$

13 Bonar R, Mohammed S, Favaloro EJ. International normalized ratio monitoring of vitamin $\mathrm{K}$ antagonist therapy: comparative performance of point-of-care and laboratory-derived testing. Semin Thromb Hemost 2015;41(03):279-286

14 Bonar R, Favaloro EJ. Explaining and reducing the variation in inter-laboratory reported values for international normalised ratio. Thromb Res 2017;150:22-29

15 Baluwala I, Favaloro EJ, Pasalic L. Therapeutic monitoring of unfractionated heparin - trials and tribulations. Expert Rev Hematol 2017;10(07):595-605

16 Kershaw G. Performance of Activated Partial Thromboplastin Time (APTT): determining reagent sensitivity to factor deficiencies, heparin, and lupus anticoagulants. Methods Mol Biol 2017; 1646:75-83

17 Stufano F, Boscarino M, Bucciarelli P, et al. Utility of von Willebrand factor propeptide in the differential diagnosis of von Willebrand disease and acquired von Willebrand syndrome. Semin Thromb Hemost 2019;45(01):36-42

18 Favaloro EJ. Diagnosis or exclusion of von Willebrand disease using laboratory testing. Methods Mol Biol 2017; 1646:391-402

19 Dorgalaleh A, Tabibian S, Shams M, et al. A unique factor XIII mutation in southeastern Iran with an unexpectedly high prevalence: Khash factor XIII. Semin Thromb Hemost 2019;45(01): 43-49

20 Alavi SER, Jalalvand M, Assadollahi V, Tabibian S, Dorgalaleh A. Intracranial hemorrhage: a devastating outcome of congenital bleeding disorders-prevalence, diagnosis, and management, with a special focus on congenital factor XIII deficiency. Semin Thromb Hemost 2018;44(03):267-275

21 Carcao M, Fukutake K, Inbal A, et al. Developing the first recombinant factor XIII for congenital factor XIII deficiency: clinical challenges and successes. Semin Thromb Hemost 2017;43(01): 59-68

22 Arachchillage DRJ, Makris M. Inherited thrombophilia and pregnancy complications: should we test? Semin Thromb Hemost 2019;45(01):50-60

23 Kubisz P, Holly P, Stasko J. Sticky platelet syndrome - 35 years of growing evidence. Semin Thromb Hemost 2019;45(01):61-68

24 Favaloro EJ, Lippi G. Commentary: controversies in thrombosis and hemostasis part 2 - does sticky platelet syndrome exist? Semin Thromb Hemost 2019;45(01):69-72

25 Huang JY, Soeharto DA, Damara I, Ignjatovic V, Linden MD, Monagle P. Antiplatelet therapy monitoring in neonates and children. Semin Thromb Hemost 2019;45(01):73-85

26 Laridan E, Martinod K, De Meyer SF. Neutrophil extracellular traps in arterial and venous thrombosis. Semin Thromb Hemost 2019; 45(01):86-93 
27 Maclachlan KH, Stevens HP, Tran HA, Chunilal SD. Weight-based enoxaparin for venous thromboembolism in obesity gives similar anti-Xa levels to patients $<100 \mathrm{~kg}$, with no increase in major bleeding. Semin Thromb Hemost 2019;45(01):94-99

28 McCaughan GJB, Favaloro EJ, Pasalic L, Curnow J. Anticoagulation at the extremes of body weight: choices and dosing. Expert Rev Hematol 2018;11(10):817-828

29 Thachil J. The elusive diagnosis of disseminated intravascular coagulation: does a diagnosis of DIC exist anymore? Semin Thromb Hemost 2019;45(01):100-107

30 Sridharan M, Coon L, Chen D, Pruthi RK, Factor V. Deficiency with a thrombotic clinical phenotype. Semin Thromb Hemost 2019;45 (01):108-112
31 Hoirisch-Clapauch S. Comment on "Venous and Arterial Thromboses: two sides of the same coin?" Semin Thromb Hemost 2019; 45(01):113-114

32 Lippi G, Favaloro EJ. Myocardial infarction, unstable angina and white thrombi: time to move forward? Semin Thromb Hemost 2019;45(01):115-116

33 Stanciakova L, Kubisz P, Stasko J, Maly J. Meeting report: The XXVth Slovak-Czech Conference on Hemostasis and Thrombosis (with international participation), Martin, Slovak Republic. Semin Thromb Hemost 2019;45(01):117-120

34 Welcome to Seminars in Thrombosis \& Hemostasis. New (2017) Impact Factor and most highly cited papers. Semin Thromb Hemost 2019;45(01):1-4 